\title{
ON SYMMETRIC NEIGHBORHOOD SYSTEMS IN METRIC, STRONGLY PARACOMPACT AND SOME OTHER TYPES OF SPACES
}

\author{
BY \\ MARGARET REAMES WISCAMB
}

I. Introduction. A mapping $U$ from a set $R$ to the set $2^{R}$ of all its subsets, written as $\{U(p) \mid p \in R\}$, is "symmetric" if $q \in U(p)$ implies $p \in U(q)$; we then refer to it as a "symmetric collection" of subsets of $R$. It is said to be finite if the collection of distinct sets $U(p)$ is finite; and similarly for star finite, locally countable, etc. Equivalently, we choose $R^{\prime} \subset R$ so that each $U(p)=U\left(p^{\prime}\right)$ for just one $p^{\prime} \in R^{\prime}$, and say that $\{U(p) \mid p \in R\}$ is finite, or star finite, etc., provided that $\left\{U(p) \mid p \in R^{\prime}\right\}$ has the corresponding property. Throughout this paper we assume $p \in U(p)$ unless the contrary is explicitly stated. An example of a symmetric collection of sets in a metric space $R$ is the collection of all spherical neighborhoods $S(p, r), p \in R$, with a fixed radius $r$.

Another less obvious example is the set of stars of points $p \in R$ by a covering $\mathscr{U}$. If $\mathscr{U}$ is a covering of $R$, then the star of $p$ by $\mathscr{U}$ is the union of all the sets of $\mathscr{U}$ which contain $p$. In symbols we write $S(p, \mathscr{U})=\bigcup\{U \mid U \in \mathscr{U} ; p \in U\}$. If $q \in S(p, \mathscr{U})$, then $p$ and $q$ are both contained in some set $U$ of $\mathscr{U}$, thus $p \in S(q, \mathscr{U})$.

In this paper we examine the effects of this property of symmetry on other known topological properties. For example, in a symmetric collection point finiteness is equivalent to star finiteness (Lemma 3.2). Using this property we obtain a number of equivalent conditions for metrizability, strong metrizability, the star finite property (strong paracompactness) and complete paracompactness.

In this paper "regular" means "regular and $T_{1}$ ", and neighborhoods and neighborhood bases are not restricted to open sets.

II. Some metrization theorems. A collection $\mathscr{U}$ of subsets of a topological space $R$ is locally finite (countable) if every $p \in R$ has a neighbourhood which meets only finitely (countable) many elements of $\mathscr{U}$. It is point finite (countable) if every point of $R$ is contained in at most finitely (countably) many sets of $\mathscr{U}$.

A Hausdorff space is said to be paracompact if every open covering of $R$ has an open locally finite refinement.

A space is fully normal if every open covering $\mathscr{U}$ has an open $\Delta$-refinement $\mathscr{V}$, that is, an open covering for which the $\operatorname{stars} S(p, \mathscr{V})$ form a covering of $R$ which refines $\mathscr{U}$.

Received by the editors February 14, 1968. 
Stone [15, Theorems 1,2] has proved that the concepts of paracompactness and full normality are equivalent.

A collection of sets $\mathscr{U}=\left\{U_{\alpha} \mid \alpha \in A\right\}$ is said to be closure preserving if for any subset $A^{\prime}$ of $A$ we have

$$
\bigcup\left\{\mathrm{Cl}\left(U_{\alpha}\right) \mid \alpha \in A^{\prime}\right\}=\mathrm{Cl}\left(\bigcup\left\{U_{\alpha} \mid \alpha \in A^{\prime}\right\}\right) .
$$

It is not hard to show that a locally finite collection is closure preserving.

A space has a $\sigma$-closure preserving base if it has a base which consists of countably many closure preserving families.

Let $\mathscr{P}$ be a collection of ordered pairs, $P=\left(P_{1}, P_{2}\right)$, of subsets of a topological space $R$ with $P_{1} \subset P_{2}$ for all $P \in \mathscr{P}$. Then $\mathscr{P}$ is called a pair-base for $R$ if $P_{1}$ is open for all $P \in \mathscr{P}$, and if, for any $p \in R$ and neighborhood $U$ of $p$, there exists a $P \in \mathscr{P}$ such that $p \in P_{1} \subset P_{2} \subset U . \mathscr{P}$ is called cushioned if for every subcollection $\mathscr{P}^{\prime}$ of $\mathscr{P}$ we have

$$
\mathrm{Cl}\left(\bigcup\left\{P_{1} \mid P \in \mathscr{P}^{\prime}\right\}\right) \subset \bigcup\left\{P_{2} \mid P \in \mathscr{P}^{\prime}\right\} .
$$

$\mathscr{P}$ is called $\sigma$-cushioned if it consists of countably many cushioned subcollections.

Ceder has investigated regular spaces with $\sigma$-closure preserving bases ( $M_{1}$ spaces), and $T_{1}$ spaces with $\sigma$-cushioned pair-bases ( $M_{3}$ spaces), and has shown that neither need be metrizable [1, Example 9.2]. We can show that if the sets of the basis form a symmetric collection, then these spaces are metrizable. To obtain these results, we use the following theorem due to Nagata [9, Theorem 1].

TheOREM 2.1 (NAGATA). In order that a $T_{1}$-topological space $R$ be metrizable it is necessary and sufficient that one can assign a neighborhood basis $\left\{U_{n}(p) \mid n=1,2, \ldots\right\}$ for every point $p$ of $R$ such that for every $n$ and each point $p$ of $R$ there exist neighborhoods $S_{n}^{1}(p), S_{n}^{2}(p)$ of p satisfying

(i) $q \notin U_{n}(p)$ implies $S_{n}^{2}(q) \cap S_{n}^{1}(p)=\varnothing$,

(ii) $q \in S_{n}^{1}(p)$ implies $S_{n}^{2}(q) \subset U_{n}(p)$.

THEOREM 2.2. A $T_{1}$ space $R$ is metrizable if and only if it has neighborhood bases $\left\{U_{n}(p) \mid n=1,2, \ldots\right\}, p \in R ;\left\{V_{n}(p) \mid n=1,2, \ldots\right\}, p \in R$, such that

(a) $V_{n}(p) \subset U_{n}(p)$ and $\left\{\left(V_{n}(p), U_{n}(p)\right) \mid p \in R\right\}$ is cushioned for each $n$ and

(b) the collections $\left\{U_{n}(p) \mid p \in R\right\}$ and $\left\{V_{n}(p) \mid p \in R\right\}$ are symmetric for each $n$.

Proof. First we prove the sufficiency. Since $\left\{\left(V_{n}(p), U_{n}(p)\right) \mid p \in R\right\}$ is cushioned for each $n$, we have

$$
\mathrm{Cl}\left(\bigcup\left\{V_{n}(p) \mid p \in P \subset R\right\}\right) \subset \bigcup\left\{U_{n}(p) \mid p \in P \subset R\right\} .
$$

Set $S_{n}^{1}(p)=R-\bigcup\left\{V_{n}(r) \mid p \notin U_{n}(r)\right\}$.

This is a neighborhood of $p$ since

$$
\begin{aligned}
p \in R-\bigcup\left\{U_{n}(r) \mid p \notin U_{n}(r)\right\} & \subset R-\mathrm{Cl}\left(\bigcup\left\{V_{n}(r) \mid p \notin U_{n}(r)\right\}\right) \\
& \subset R-\bigcup\left\{V_{n}(r) \mid p \notin U_{n}(r)\right\} .
\end{aligned}
$$

Set $S_{n}^{2}(p)=V_{n}(p)$ 
It is easy to see that these sets satisfy the conditions of the theorem.

THEOREM 2.3. A regular space $R$ is metrizable if and only if there exist neighborhood bases $\left\{U_{n}(p) \mid n=1,2, \ldots\right\}, p \in R$, such that

(a) $\mathscr{U}_{n}=\left\{U_{n}(p) \mid p \in R\right\}$ is closure preserving, and

(b) $p \in \mathrm{Cl}\left(U_{n}(q)\right)$ implies $q \in \mathrm{Cl}\left(U_{n}(p)\right)$.

Proof. The condition is sufficient. Since $R$ is regular, $\left\{\mathrm{Cl}\left(U_{n}(p)\right) \mid n=1,2, \ldots\right\}$ forms a neighborhood basis at $p$.

Set $S_{n}^{1}(p)=R-\bigcup\left\{\mathrm{Cl}\left(U_{n}(r)\right) \mid p \notin \mathrm{Cl}\left(U_{n}(r)\right)\right\}, S_{n}^{2}(p)=\mathrm{Cl}\left(U_{n}(p)\right)$.

Since $\mathscr{U}_{n}$ is closure preserving for every $n, S_{n}^{1}(p)$ is an open set containing $p$. We must show that (i) $q \notin \mathrm{Cl}\left(U_{n}(p)\right)$ implies $S_{n}^{2}(q) \cap S_{n}^{1}(p)=\varnothing$.

(1) $q \notin \mathrm{Cl}\left(U_{n}(p)\right)$ implies $p \notin \mathrm{Cl}\left(U_{n}(q)\right)$ by (b).

Suppose there is a point $x$ in $S_{n}^{2}(q) \cap S_{n}^{1}(p)$. Then $x \in S_{n}^{2}(q)=\mathrm{Cl}\left(U_{n}(q)\right) . x \in S_{n}^{1}(p)$ implies $x \notin \bigcup\left\{\mathrm{Cl}\left(U_{n}(r)\right) \mid p \notin \mathrm{Cl}\left(U_{n}(r)\right)\right\}$, that is, $p \notin \mathrm{Cl}\left(U_{n}(r)\right)$ implies $x \notin \mathrm{Cl}\left(U_{n}(r)\right)$. But $p \notin \mathrm{Cl}\left(U_{n}(q)\right)$ by (1). This implies $x \notin \mathrm{Cl}\left(U_{n}(q)\right)$, which is a contradiction. Thus $S_{n}^{2}(p) \cap S_{n}^{1}(q)=\varnothing$.

Now we show that (ii) $q \in S_{n}^{1}(p)$ implies $S_{n}^{2}(q) \subset \mathrm{Cl}\left(U_{n}(p)\right)$. If $q \in S_{n}^{1}(p)$, then $q \notin \bigcup\left\{\mathrm{Cl}\left(U_{n}(r)\right) \mid p \notin \mathrm{Cl}\left(U_{n}(r)\right)\right\}$, that is,

(2) $p \notin \mathrm{Cl}\left(U_{n}(r)\right)$ implies $q \notin \mathrm{Cl}\left(U_{n}(r)\right)$.

Suppose $x \notin \mathrm{Cl}\left(U_{n}(p)\right)$. Then $p \notin \mathrm{Cl}\left(U_{n}(x)\right)$ by (b). From (2) we have $q \notin \mathrm{Cl}\left(U_{n}(x)\right)$ which implies $x \notin \mathrm{Cl}\left(U_{n}(q)\right)=S_{n}^{2}(q)$. Thus (ii) holds, and $R$ is metrizable by Theorem 2.1.

Next we prove the necessity. Let $R$ be a metric space. Then $\mathscr{V}_{n}=\{S(p, 1 / n) \mid p \in R\}$ is an open covering of $R$ for each $n$. Since $R$ is paracompact, $\mathscr{V}_{n}$ has a closed locally finite refinement $\mathscr{U}_{n}[4$, Lemma 1$]$.

We note that for each $n$ and $p, S\left(p, \mathscr{U}_{n}\right)$ is a neighborhood of $p$, for $p \in R$ $-\bigcup\left\{U \mid U \in \mathscr{U}_{n}, p \notin U\right\} \subset S\left(p, \mathscr{U}_{n}\right)$, and $R-\bigcup\left\{U \mid U \in \mathscr{U}_{n}, p \notin U\right\}$ is open by the local finiteness of $\mathscr{U}_{n}$. Since $\mathscr{U}_{n}$ refines $\mathscr{V}_{n}$,

$$
S\left(p, \mathscr{U}_{n}\right) \subset S\left(p, \mathscr{V}_{n}\right)=\bigcup\{S(q, 1 / n) \mid p \in S(q, 1 / n)\} \subset S(p, 2 / n) .
$$

Thus $\left\{S\left(p, \mathscr{U}_{n}\right) \mid n=1,2, \ldots\right\}$ is a neighborhood basis at $p$. From the local finiteness of $\mathscr{U}_{n}$ and the fact that $U \in \mathscr{U}_{n}$ implies $U$ is closed, we have $q \in \mathrm{Cl}\left(S\left(p, \mathscr{U}_{n}\right)\right)$ implies $p \in \mathrm{Cl}\left(S\left(q, \mathscr{U}_{n}\right)\right)$.

Since $\mathscr{U}_{n}$ is closure preserving $\mathscr{S}_{n}=\left\{S\left(p, \mathscr{U}_{n}\right) \mid p \in R\right\}$ is closure preserving also.

III. The star finite property. A covering $\mathscr{U}$ of a topological space $R$ is said to be star finite (countable) if each set of $\mathscr{U}$ meets only finitely (countably) many other sets of $\mathscr{U}$.

If every open covering of $R$ has an open star finite refinement, then we say that $R$ has the star finite property, or $R$ is strongly paracompact.

Michael [4, Lemma 1] has proved that in the definition of paracompactness the word "open" used in referring to the refinement may be replaced by the word 
“closed", or even deleted completely. This does not hold for the star finite property, for any $T_{1}$ space $R$ has the property that every open covering has a closed star finite refinement. We can take the refinement to be $\{\{p\} \mid p \in R\}$.

However, we can obtain results similar to Michael's if we adopt suitable restrictions. For this see §II(iv), (v), and (vi) of Theorem 3.1.

THEOREM 3.1. For a regular space $R$, the following are equivalent:

I. $R$ has the star finite property.

II. Every open covering of $R$ has a refinement $\mathscr{U}=\{U(p) \mid p \in R\}$ where $\mathscr{U}$ is respectively

(i) a symmetric star finite collection of open sets, or

(ii) a symmetric locally finite collection of open sets, or

(iii) a symmetric point finite collection of open sets, or

(iv) a symmetric locally finite collection of (not necessarily open) sets and each $U(p)$ is a neighborhood of $p$, or

(v) a (not necessarily symmetric) locally finite collection of sets satisfying $q \in \mathrm{Cl}(U(p))$ implies $p \in \mathrm{Cl}(U(q))$, or

(vi) a symmetric locally finite collection of closed sets, or

(vii), (viii), (ix) a symmetric collection of open sets which is respectively (vii) star-countable, (viii) locally countable, (ix) point-countable.

III. Every open covering of $R$ has an open star countable refinement.

Proof. The proof will proceed as follows:

$\mathrm{I} \Rightarrow \mathrm{II}(\mathrm{i}) \Rightarrow \mathrm{II}(\mathrm{ii}) \Rightarrow \mathrm{II}(\mathrm{iii}) \Rightarrow \mathrm{II}(\mathrm{iv}) \Rightarrow \mathrm{II}(\mathrm{vi}) \Rightarrow \mathrm{I}$;

$\mathrm{II}(\mathrm{v}) \Leftrightarrow \mathrm{II}(\mathrm{vi})$; II(i) $\Rightarrow \mathrm{II}($ vii) $\Rightarrow \mathrm{II}($ viii $) \Rightarrow \mathrm{II}(\mathrm{ix}) \Rightarrow \mathrm{III} \Rightarrow \mathrm{I}$.

$\mathrm{I} \Rightarrow \mathrm{II}(\mathrm{i})$. Let $\mathscr{W}$ be an arbitrary open covering of $R$. Since $R$ is paracompact, $\mathscr{W}$ has an open $\Delta$-refinement, $\mathscr{U}$. By hypothesis, $\mathscr{U}$ has an open star finite refinement $\mathscr{V}$.

Then $\mathscr{S}=\{S(p, \mathscr{V}) \mid p \in R\}$ is an open star finite covering of $R$, and $S(p, \mathscr{V})$ $\subset S(p, \mathscr{U}) \subset W$ for some $W \in \mathscr{W}$. Hence $\mathscr{S}$ refines $\mathscr{W}$. Moreover, $q \in S(p, \mathscr{V})$ implies $p \in S(q, \mathscr{V})$. Thus II(i) holds.

$\mathrm{II}(\mathrm{i}) \Rightarrow \mathrm{II}(\mathrm{ii}) \Rightarrow \mathrm{II}(\mathrm{iii})$ is obvious.

II(iii) $\Rightarrow$ II(iv). This follows from the following lemma:

LEMMA 3.2. A symmetric point finite collection $\mathscr{U}=\{U(p) \mid p \in R\}$ is star finite.

Proof. Let $U\left(p_{0}\right)$ be an arbitrary set of $\mathscr{U}$. The collection $\left\{U(p) \mid p \in U\left(p_{0}\right)\right\}$ contains only finitely many distinct elements of $\mathscr{U}$, say $U\left(p_{1}\right), U\left(p_{2}\right), \ldots, U\left(p_{k}\right)$. This follows from the point finiteness of $\mathscr{U}$ and the fact that $p \in U\left(p_{0}\right)$ implies $p_{0} \in U(p)$.

Similarly, for $i=1,2, \ldots, k$, the collection $\left\{U(p) \mid p \in U\left(p_{i}\right)\right\}$ contains only finitely many distinct elements, say $U\left(p_{i, 1}\right), U\left(p_{i, 2}\right), \ldots, U\left(p_{i, r(i)}\right)$.

Let us denote by $\mathscr{U}\left(p_{0}\right)$ the finite collection $\left\{U\left(p_{i, j}\right) \mid j=1,2, \ldots, r(i)\right.$; $i=1,2, \ldots, k\}$. 
Now suppose $U(q) \cap U\left(p_{0}\right) \neq \varnothing$. Then there exists an $r \in U(q) \cap U\left(p_{0}\right)$. $r \in U\left(p_{0}\right)$ implies $U(r)=U\left(p_{i}\right)$ for some $i$. Since $r \in U(q)$ then $q \in U(r)=U\left(p_{i}\right)$, hence $U(q)=U\left(p_{i, j}\right)$ for some $j$. Thus $U(q)$ is an element of the finite collection $\mathscr{U}\left(p_{0}\right)$ and $\mathscr{U}$ is star finite.

We now prove II(iii) $\Rightarrow$ II(iv).

Let $\mathscr{W}$ be an arbitrary open covering of $R$. $\mathscr{W}$ has an open symmetric point finite refinement $\mathscr{U}=\{U(p) \mid p \in R\}$, and by Lemma 3.2, $\mathscr{U}$ is star finite. Since each set of $\mathscr{U}$ is open, $\mathscr{U}$ is locally finite as well, hence II(iv) holds.

$\mathrm{II}(\mathrm{iv}) \Rightarrow \mathrm{II}(\mathrm{vi})$. We first prove a lemma.

LEMMA 3.3. If $\mathscr{U}=\{U(p) \mid p \in R\}$ is a symmetric point finite collection of sets, where $U(p)$ is a neighborhood of $p$, then $\{\mathrm{Cl}(U(p)) \mid p \in R\}$ is star finite.

In the proof we use the following (standard) notation. The star of a set $A$ by a covering $\mathscr{U}$ is the union of all the sets of $\mathscr{U}$ which meet $A$. In symbols, $S(A, \mathscr{U})$ $=\bigcup\{U \mid U \in \mathscr{U} ; U \cap A \neq \varnothing\}$. Since $S(A, \mathscr{U})$ is a set, we may speak of the star of $S(A, \mathscr{U})$ by $\mathscr{U}$, and we write $S(S(A, \mathscr{U}), \mathscr{U})=S^{2}(A, \mathscr{U})$. Thus inductively we may define $S^{n}(A, \mathscr{U})$ for any positive integer $n$.

From Lemma 3.2 it follows that $\{U(q) \mid q \in S(U(p), \mathscr{U})\}$ is a finite collection. In a similar manner, it follows that $\left\{U(q) \mid q \in S^{n}(U(p), \mathscr{U})\right\}$ is a finite collection for every positive integer $n$.

Suppose $\mathrm{Cl}(U(p)) \cap \mathrm{Cl}(U(q)) \neq \varnothing$. Then there exists an $r$ such that $U(r)$ contains a point $x$ of $U(p)$ and a point $y$ of $U(q)$. Hence $U(q) \in\left\{U(q) \mid q \in S^{2}(U(p), \mathscr{U})\right\}$ which is a finite set.

We now return to the proof of II(iv) $\Rightarrow \mathrm{II}(\mathrm{vi})$.

Let $\mathscr{W}$ be an arbitrary open covering of $R$. Since $R$ is paracompact, $\mathscr{W}$ has an open $\Delta$-refinement $\mathscr{V}$. Since $R$ is regular, $R$ has an open covering $\mathscr{U}$ such that the collection of closures of sets of $\mathscr{U}$ refines $\mathscr{V}$.

By hypothesis, $\mathscr{U}$ has a symmetric locally finite refinement $\mathscr{U}^{\prime}=\left\{U^{\prime}(p) \mid p \in R\right\}$. By the lemma, $\mathrm{Cl}\left(\mathscr{U}^{\prime}\right)=\left\{\mathrm{Cl}\left(U^{\prime}(p)\right) \mid p \in R\right\}$ is star finite.

Put $\mathscr{S}=\left\{S\left(p, \mathrm{Cl}\left(\mathscr{U}^{\prime}\right) \mid p \in R\right\}\right.$. Then $\mathscr{S}$ is a symmetric locally finite collection of closed sets and $\mathscr{S}$ refines $\mathscr{W}$.

$\mathrm{II}(\mathrm{vi}) \Rightarrow \mathrm{I}$. Let $\mathscr{W}$ be an arbitrary open covering of $R$. By hypothesis, $\mathscr{W}$ has a closed symmetric locally finite refinement $\mathscr{U}=\{U(p) \mid p \in R\}$. This implies $\mathscr{U}$ is star finite by Lemma 3.2.

Set $V(p)=R-\bigcup\{U(q) \mid p \notin U(q)\}$. Then $\mathscr{V}=\{V(p) \mid p \in R\}$ is an open covering of $R$ by the local finiteness of $\mathscr{U}$. Then $V(p)=\{x \mid U(x) \subset U(p)\}$.

From this it is easy to see that $V(p) \subset U(p)$, hence $\mathscr{V}$ refines $\mathscr{W}$. Moreover it is clear that $\mathscr{V}$ is star finite since $\mathscr{U}$ is. Hence $R$ has the star finite property.

$\mathrm{II}(\mathrm{vi}) \Rightarrow \mathrm{II}(\mathrm{v})$ is obvious.

$\mathrm{II}(\mathrm{v}) \Rightarrow \mathrm{II}(\mathrm{vi})$. Let $\mathscr{W}$ be an arbitrary open covering of $R$. Since $R$ is regular, $R$ has an open covering $\mathscr{V}$ such that the collection of closures of $\mathscr{V}$ refines $\mathscr{W}$.

By hypothesis, $\mathscr{V}$ has a locally finite refinement $\mathscr{U}=\{U(p) \mid p \in R\}$ such that $q \in \mathrm{Cl}(U(p))$ implies $p \in \mathrm{Cl}(U(q))$. 
Then $\mathrm{Cl}(\mathscr{U})=\{\mathrm{Cl}(U(p)) \mid p \in R\}$ is also locally finite and $\mathrm{Cl}(U(p)) \subset \mathrm{Cl}(V) \subset W$ for some $W \in \mathscr{W}$. Hence II(vi) is satisfied.

$\mathrm{II}(\mathrm{i}) \Rightarrow \mathrm{II}($ vii) $\Rightarrow \mathrm{II}($ viii $) \Rightarrow \mathrm{II}(\mathrm{ix})$ is evident.

II(ix) $\Rightarrow$ III. A symmetric point countable collection $\mathscr{U}=\{U(p) \mid p \in R\}$ is star countable. The proof of this is the same as that of Lemma 3.2, mutatis mutandis.

III $\Rightarrow$ I. This result has been obtained by Smirnov [14]. We sketch the proof, for completeness.

Let $\mathscr{W}$ be an arbitrary open covering of $R$. By hypothesis, $\mathscr{W}$ has an open star countable refinement, $\mathscr{U}=\left\{U_{\beta} \mid \beta \in B\right\}$.

Since $\left\{U_{\beta} \mid U_{\beta} \subset S^{n}\left(U_{\alpha}, \mathscr{U}\right)\right\}, n=1,2, \ldots$ is countable, we may assume that $\mathscr{U}$ consists of open sets, $U_{\gamma, i}, \gamma \in \Gamma, i=1,2, \ldots$ such that $U_{\gamma, i} \cap U_{\delta, j}=\varnothing$ for $\gamma \neq \delta$.

Put $U_{\gamma}=\bigcup_{i=1}^{\infty} U_{\gamma, i}$. Then $U_{\gamma}$ is both open and closed in $R$. If we choose one set $U_{\gamma, i}$ for each $\gamma \in \Gamma$, this will be a discrete collection and countably many such collections make up $\mathscr{U}$. Thus $\mathscr{U}$ is a $\sigma$-discrete refinement of $\mathscr{W}$, and $R$ is paracompact.

Since $R$ is paracompact $U_{\gamma}$ is paracompact for $\gamma \in \Gamma$, and $\left\{U_{\gamma, i} \mid i=1,2, \ldots\right\}$ is a countable open covering of $U_{\gamma}$. Then by a theorem of Morita [7, Theorem 3] $\left\{U_{\gamma, i} \mid i=1,2, \ldots\right\}$ has an open star finite refinement, $\mathscr{V}_{\gamma}$. The collection of all such refinements for $\gamma \in \Gamma$ is an open star finite refinement of $\mathscr{U}$, hence of $\mathscr{W}$. Thus $R$ has the star finite property.

IV. Strongly metrizable spaces. We recall Nagata's [11, Theorem 1] characterization of a metric space. A regular space is metrizable if and only if it has a base consisting of countably many locally finite open coverings. A regular space $R$ is said to be strongly metrizable if it has a base which consists of countably many star finite open coverings. We say such a basis is $\sigma$-star finite.

In Theorem 4.1 we characterize strongly metrizable spaces by a number of equivalent conditions, some of which appear to be considerably weaker than this definition, while one, (ii), appears to be stronger.

THEOREM 4.1. For a regular space $R$, the following are equivalent:

I. $R$ has a $\sigma$-star finite open basis (i.e., is strongly metrizable).

II. There exist neighborhood bases $\left\{U_{n}(p) \mid n=1,2, \ldots\right\}, p \in R$, with $\mathscr{U}_{n}$ $=\left\{U_{n}(p) \mid p \in R\right\}$ where $\mathscr{U}_{n}$ is respectively

(i) a symmetric star finite collection of open sets, or

(ii) a symmetric locally finite collection of open sets, or

(iii) a symmetric point finite collection of open sets, or

(iv) a symmetric locally finite collection of (not necessarily open) sets, or

(v) a symmetric locally finite collection of closed sets, or

(vi), (vii), (viii) a symmetric collection of open sets which is respectively (vi) star countable, (vii) locally countable, or (viii) point countable.

III. $R$ has a $\sigma$-star countable open basis. 
The proof proceeds as follows: $\mathrm{I} \Rightarrow \mathrm{II}(\mathrm{i}) \Rightarrow \mathrm{II}(\mathrm{ii}) \Rightarrow \mathrm{II}(\mathrm{iii}) \Rightarrow \mathrm{II}(\mathrm{iv}) \Rightarrow \mathrm{II}(\mathrm{v})$; $\mathrm{II}(\mathrm{i}) \Rightarrow \mathrm{II}($ vi $) \Rightarrow \mathrm{II}($ vii) $\Rightarrow \mathrm{II}($ viii $) \Rightarrow \mathrm{III} \Rightarrow \mathrm{I}$.

I $\Rightarrow$ II(i). By hypothesis, $R$ has a basis $\mathscr{U}=\bigcup_{n=1}^{\infty} \mathscr{U}_{n}, \mathscr{U}_{n}=\left\{U_{n, \beta} \mid \beta \in B_{n}\right\}$, where $\mathscr{U}_{n}$ is a star finite open covering of $R$. Since $R$ is metrizable it is perfectly normal, hence we can write $R-U_{n, \beta}=\bigcap_{k=1}^{\infty} V_{n, \beta}^{(k)}$, where the $V_{n, \beta}^{(k)}$ are open sets.

Let $\Delta$ be a finite subset of $B_{n}$. Put

$$
N_{n, \Delta}^{(k)}=\left[\bigcap_{\beta \in \Delta} U_{n, \beta}\right] \cap\left[\bigcap_{\beta \notin \Delta} V_{n, \beta}^{(k)}\right] .
$$

$N_{n, \Delta}^{(k)}$ is open. This follows from the star finiteness of $\mathscr{U}_{n}$, for $R-U_{n, \beta} \subset V_{n, \beta}^{(k)}$ implies $R-V_{n, \beta}^{(k)} \subset U_{n, \beta}$. Thus $\left\{R-V_{n, \beta}^{(k)} \mid \beta \in B_{n}\right\}$ is star finite also and closure preserving. Therefore $\bigcup_{\beta \notin \Delta}\left(R-V_{n, \beta}^{(k)}\right)$ is closed, and $R-\bigcup_{\beta \notin \Delta}\left(R-V_{n, \beta}^{i k)}\right)=\bigcap_{\beta \notin \Delta} V_{n, \beta}^{(k)}$ is open. Since $\Delta$ is a finite set, it follows that $N_{n, \Delta}^{(k)}$ is open.

$\mathscr{R}_{n}^{(k)}=\left\{N_{n, \Delta}^{(k)} \mid \Delta \subset B_{n} ; \Delta\right.$ finite $\}$ is a star finite covering of $R$.

To see that it is a covering, let $p$ be arbitrary in $R$. Then $p \in \bigcap_{\beta \in \Delta} U_{n, \beta}$ for some finite subset $\Delta$ of $B_{n}$, and $p \notin U_{n, \beta}$ for $\beta \notin \Delta$. Then $p \in V_{n, \beta}^{(k)} ; k=1,2, \ldots, \beta \notin \Delta$, hence $p \in N_{n, \Delta}^{(k)}$.

To show $\mathscr{K}_{n}^{(k)}$ is star finite, let $N_{n, \Delta}^{(k)}$ be a set of $\mathscr{R}_{r .}^{(k)}$. Then for $\beta_{0} \in \Delta, N_{n, \Delta}^{(k)} \subset U_{n, \beta_{0}}$. Suppose

(1) $N_{n, \Delta}^{(k)} \cap N_{n, \Delta^{\prime}}^{(k)} \neq \varnothing$. Then

(2) $U_{n, \beta_{0}} \cap N_{n, \Delta^{\prime}}^{(k)} \neq \varnothing$.

By the construction of $N_{n, \Delta^{\prime}}^{(k)}$, this implies that $\Delta^{\prime}$ consists of those indices $\beta^{\prime}$ for which $U_{n, \beta_{0}} \cap U_{n, \beta} \neq \varnothing$. Since this is true for only finitely many $\beta^{\prime}$, then only finitely many $\Delta^{\prime}$ can satisfy (2), hence only finitely many $N_{n, \Delta^{\prime}}^{(k)}$ satisfy (1).

Next we show that for every open set $G$ and each point $p \in G$ there exists an $n$ and a $k$ such that $S\left(p, \mathscr{R}_{n}^{(k)}\right) \subset G$. Since $\mathscr{U}$ is a basis for $R$, there exists an $n$ and a $\beta_{0}$ such that $p \in U_{n, \beta_{0}} \subset G$. Also there exists a $\Delta_{0}$ such that $p \in U_{n, \beta}$ for $\beta \in \Delta_{0}$, and $p \notin U_{n, \beta}$ for $\beta \notin \Delta_{0}$. Then $\beta_{0} \in \Delta_{0}$.

Since $p \in U_{n, \beta_{0}}, p \notin R-U_{n, \beta_{0}}=\bigcap_{k=1}^{\infty} V_{n, \beta_{0}}^{(k)}$. Thus there exists a $k$ such that $p \notin V_{n, \beta_{0}}^{(k)}$. For this $n$ and $k$, let us consider $\mathscr{K}_{n}^{(k)}$. If $p \in N_{n, \Delta}^{(k)}$ then since $p \notin V_{n, \beta_{0}}^{(k)}$, we must have $\beta_{0} \in \Delta$. Hence $N_{n, \Delta}^{(k)} \subset U_{n, \beta_{0}}$, i.e., $S\left(p, \mathscr{R}_{n}^{(k)}\right) \subset U_{n, \beta_{0}} \subset G$.

Thus $\left\{S\left(p, \mathscr{R}_{n}^{(k)}\right) \mid n=1,2, \ldots ; k=1,2, \ldots\right\}$ is a neighborhood basis at $p$. Moreover $\mathscr{S}_{n, k}=\left\{S\left(p, \mathscr{K}_{n}^{(k)}\right) \mid p \in R\right\}$ is star finite since $\mathscr{K}_{n}^{(k)}$ is, and it is symmetric. This proves II(i).

$\mathrm{II}(\mathrm{i}) \Rightarrow \mathrm{II}(\mathrm{ii}) \Rightarrow \mathrm{II}(\mathrm{iii})$ is obvious.

II(iii) $\Rightarrow$ II(iv). By Lemma 3.2, $\mathscr{U}_{n}$ is star finite. Since by hypothesis its sets are open, then $\mathscr{U}_{n}$ is also locally finite. Thus II(iv) holds.

$\mathrm{II}(\mathrm{iv}) \Rightarrow \mathrm{II}(\mathrm{v})$. By hypothesis, $R$ has neighborhood bases $\left\{U_{n}(p) \mid n=1,2, \ldots\right\}$ such that $\mathscr{U}_{n}$ is locally finite and symmetric. Since $R$ is regular, $\left\{\mathrm{Cl}\left(U_{n}(p)\right) \mid n=1,2, \ldots\right\}$ is a neighborhood basis at $p$. By Lemma $3.3, \mathrm{Cl}\left(\mathscr{U}_{n}\right)=\left\{\mathrm{Cl}\left(U_{n}(p)\right) \mid p \in R\right\}$ is star finite.

Put

$$
V_{n, \Delta}=\left[\bigcap_{p \notin \Delta} \mathrm{Cl}\left(U_{n}(p)\right)\right] \cap\left[\bigcap_{p \notin \Delta} \mathrm{Cl}\left(R-U_{n}(p)\right)\right]
$$


for $\Delta$ a finite subset of $R^{\prime}$, where $R^{\prime}$ is a subset of $R$ such that $p, q \in R^{\prime}, p \neq q$ implies $U_{n}(p) \neq U_{n}(q)$. Then $p \notin \Delta$ means $p \in R^{\prime}-\Delta$. $V_{n, \Delta}$ is closed.

As in the proof of $\mathrm{I} \Rightarrow \mathrm{II}(\mathrm{i})$ we can show that $\mathscr{V}_{n}=\left\{V_{n, \Delta} \mid \Delta \subset R^{\prime} ; \Delta\right.$ finite $\}$ is a star finite covering of $R$.

Since $\mathscr{V}_{n}$ is not an open collection, the fact that it is star finite does not necessarily mean that it is locally finite. $\mathscr{V}_{n}$ can easily be shown to be locally finite, however, from the local finiteness of $\mathrm{Cl}\left(U_{n}\right)$.

Suppose $p_{0} \in V_{n, \Delta}$. Then $V_{n, \Delta} \subset \mathrm{Cl}\left(U_{n}\left(p_{0}\right)\right)$. For assume $p_{0} \in R^{\prime}-\Delta$. Then $p_{0} \in V_{n, \Delta} \subset \mathrm{Cl}\left(R-U_{n}\left(p_{0}\right)\right)$, which contradicts the fact that $U_{n}\left(p_{0}\right)$ is a neighborhood of $p_{0}$. Thus $p_{0} \in \Delta$ and $V_{n, \Delta} \subset \mathrm{Cl}\left(U_{n}\left(p_{0}\right)\right)$. If $p_{0} \in R-R^{\prime}$, then there exists a $q_{0} \in R^{\prime}$ such that $U_{n}\left(p_{0}\right)=U_{n}\left(q_{0}\right)$. Then by the same reasoning, $q_{0} \in \Delta$, and $p_{0} \in V_{n, \Delta} \subset \mathrm{Cl}\left(U_{n}\left(q_{0}\right)\right)=\mathrm{Cl}\left(U_{n}\left(p_{0}\right)\right)$.

Hence $S\left(p_{0}, \mathscr{V}_{n}\right) \subset \mathrm{Cl}\left(U_{n}\left(p_{0}\right)\right)$.

Set $\mathscr{S}=\bigcup_{n=1}^{\infty} \mathscr{S}_{n} ; \mathscr{S}_{n}=\left\{S\left(p, \mathscr{V}_{n}\right) \mid p \in R\right\}$. Then $\mathscr{S}_{n}$ is a star finite and locally finite symmetric collection of closed sets and $\left\{S\left(p, \mathscr{V}_{n}\right) \mid n=1,2, \ldots\right\}$ is a neighborhood basis at $p$.

Thus II(v) holds.

$\mathrm{II}(\mathrm{v}) \Rightarrow \mathrm{I}$. By hypothesis, $R$ has a closed neighborhood basis $\left\{U_{n}(p) \mid n=1,2, \ldots\right\}$, $p \in R$, such that $\mathscr{U}_{n}=\left\{U_{n}(p) \mid p \in R\right\}$ is locally finite and symmetric. By Lemma 3.2, $\mathscr{U}_{n}$ is star finite.

Since $U_{n}(p)$ is a neighborhood of $p, p \in \operatorname{Int} U_{n}(p)$ (=Interior $U_{n}(p)$ ) and $\left\{\right.$ Int $\left.U_{n}(p) \mid p \in R\right\}$ is star finite since $\mathscr{U}_{n}$ is. Clearly $\left\{\right.$ Int $\left.U_{n}(p) \mid p \in R ; n=1,2, \ldots\right\}$ is an open basis for $R$. Thus I holds.

$\mathrm{I}(\mathrm{i}) \Rightarrow \mathrm{II}(\mathrm{vi}) \Rightarrow \mathrm{II}($ vii) $\Rightarrow \mathrm{II}($ viii) is evident.

II(viii) $\Rightarrow$ III. By the proof of II(ix) $\Rightarrow$ III in Theorem 3.1, $\mathscr{U}_{n}$ is star countable, hence III holds.

III $\Rightarrow$ I. It is known $[17$, p. 113] that a regular space $R$ has a $\sigma$-star finite basis if and only if it has a $\sigma$-star countable basis.

Clearly a strongly metrizable space is metrizable. The converse is not true. A connected strongly metrizable space $R$ must have a countable base. For let $\mathscr{U}=\bigcup_{n=1}^{\infty} \mathscr{U}_{n}$ be a $\sigma$-star finite open basis for $R$. From the connectedness of $R$ it follows that each $\mathscr{U}_{n}$ is countable, therefore $\mathscr{U}$ is a countable basis. Thus any connected nonseparable metric space provides a counterexample.

V. Complete paracompactness. We shall call a regular space completely paracompact if every open covering of $R$ has a refinement which is a subcollection of an open covering $\mathscr{U}=\bigcup_{n=1}^{\infty} \mathscr{U}_{n}$, where each $\mathscr{U}_{i}$ is an open star finite covering of $R$. This definition is due to Smirnov [14].

We shall see that for metric spaces, complete paracompactness is equivalent to strong metrizability (Corollary to Theorem 6.1).

The similarity between the definition of complete paracompactness and Michael's [4, Theorem 1] characterization of paracompactness (a regular space $R$ is paracompact if and only if every open covering of $R$ has an open refinement 
$\mathscr{U}=\bigcup_{n=1}^{\infty} \mathscr{U}_{n}$, where each $\mathscr{U}_{i}$ is a locally finite collection of open subsets of $R$ ) might lead one to speculate that the star finite property and complete paracompactness are equivalent. This is not the case. Nagata [10, p. 169] has given an example of a space which is completely paracompact but does not have the star finite property.

Zarelua [19, Theorem 3] has shown that a regular space with the star finite property is completely paracompact, and every completely paracompact space is paracompact. Nagata's example and the example on page 438 show that the converse of these statements is not true.

THEOREM 5.1. For a regular space $R$, the following are equivalent:

I. $R$ is completely paracompact.

II. Every open covering of $R$ has a refinement $\mathscr{I}^{\prime}=\bigcup_{n} \mathscr{U}_{n}^{\prime},(n=1,2, \ldots)$ with $\mathscr{V}_{n}^{\prime}=\left\{U_{n}(p) \mid p \in P_{n}\right\}$ where $\bigcup_{n} P_{n}=R$, and where each $\mathscr{U}_{n}^{\prime}$ is a subcollection of $\mathscr{U}_{n}=\left\{U_{n}(p) \mid p \in R\right\}$ where $\mathscr{U}_{n}$ is respectively

(i) a symmetric star finite collection of open sets, or

(ii) a symmetric locally finite collection of open sets, or

(iii) a symmetric point finite collection of open sets, or

(iv) a symmetric locally finite collection of (not necessarily open) sets, and each $U_{n}(p)$ is a neighborhood of $p$, or

(v) a (not necessarily symmetric) locally finite collection of sets satisfying $q \in \mathrm{Cl}\left(U_{n}(p)\right) \Rightarrow p \in \mathrm{Cl}\left(U_{n}(q)\right)$, or

(vi) a symmetric locally finite collection of closed sets, or

(vii), (viii), (ix) a symmetric collection of open sets which is respectively (vii) star countable, (viii) locally countable, or (ix) point countable.

III. Every open covering of $R$ has a refinement $\mathscr{U}^{\prime}$ which is a subcollection of $\mathscr{U}=\bigcup_{n} \mathscr{U}_{n}$ where $\mathscr{U}_{n}$ is a star countable open covering of $R$.

The proof of the equivalence of these conditions follows this pattern:

$\mathrm{I} \Rightarrow \mathrm{II}(\mathrm{i}) \Rightarrow \mathrm{II}(\mathrm{ii}) \Rightarrow \mathrm{II}(\mathrm{iii}) \Rightarrow \mathrm{II}(\mathrm{iv}) \Rightarrow \mathrm{II}(\mathrm{vi}) \Rightarrow \mathrm{I}$; II(v) $\Leftrightarrow \mathrm{II}(\mathrm{vi})$;

$\mathrm{II}(\mathrm{i}) \Rightarrow \mathrm{II}($ vii) $\Rightarrow \mathrm{II}($ viii) $\Rightarrow \mathrm{II}(\mathrm{ix}) \Rightarrow \mathrm{III} \Rightarrow \mathrm{I}$.

I $\Rightarrow$ II(i). Let $\mathscr{W}$ be an arbitrary open covering of a completely paracompact space $R$. Then $\mathscr{W}$ has an open refinement $\mathscr{U}^{\prime}$ which is a subcollection of $\mathscr{U}=\bigcup_{n=1}^{\infty} \mathscr{U}_{n} ; \mathscr{U}_{n}=\left\{U_{n, \beta} \mid \beta \in B_{n}\right\}, \mathscr{U}_{n}$ an open star finite covering of $R$. $\mathscr{U}^{\prime}$ $=\bigcup_{n=1}^{\infty} \mathscr{U}_{n}^{\prime} ; \mathscr{U}_{n}^{\prime}=\left\{U_{n, \beta} \mid \beta \in B_{n}^{\prime}\right\} ; B_{n}^{\prime} \subset B_{n}$.

Since $R$ is paracompact, by a theorem of E. Michael [5, Theorem 1], $\mathscr{U}^{\prime}$ has a closure preserving closed refinement

$$
\left\{F_{n, \beta}^{\prime} \mid \beta \in B_{n}^{\prime} ; \quad n \in\{1,2, \ldots\}\right\}
$$

such that $F_{n, \beta}^{\prime} \subset U_{n, \beta}$ for all $n$ and $\beta \in B_{n}^{\prime}$.

Put $P_{n}=\bigcup\left\{F_{n, \beta}^{\prime} \mid \beta \in B_{n}^{\prime}\right\}$. Then $\bigcup_{n=1}^{\infty} P_{n}=R$.

Similarly, $\mathscr{U}_{n}$ has a closure preserving closed refinement $\left\{F_{n, \beta} \mid \beta \in B_{n}\right\}$ such that $F_{n, \beta} \subset U_{n, \beta}$. 
We form a closed covering $\mathscr{F}_{n}$ of $R$ for every $n$ by setting

$$
\begin{aligned}
F_{n, \beta}^{\prime \prime} & =F_{n, \beta}^{\prime} \cup F_{n, \beta} & & \text { for } \beta \in B_{n}^{\prime} . \\
& =F_{n, \beta} & & \text { for } \beta \in B_{n}-B_{n}^{\prime} .
\end{aligned}
$$

Then $\mathscr{F}_{n}=\left\{F_{n, \beta}^{\prime \prime} \mid \beta \in B_{n}\right\}$ is also closure preserving and $F_{n, \beta}^{\prime \prime} \subset U_{n, \beta}$ for every $\beta \in B_{n}$. Moreover, a subcollection of $\mathscr{F}_{n},\left\{F_{n, \beta}^{\prime \prime} \mid \beta \in B_{n}^{\prime}\right\}$ covers $P_{n}$.

Put $V_{n, \Delta}=\bigcap_{\beta \in \Delta} U_{n, \beta} \cap\left[\bigcap_{\beta \notin \Delta}\left(R-F_{n, \beta}^{\prime \prime}\right)\right]$ for $\Delta$ a finite subset of $B_{n}$. Then $V_{n, \Delta}=\bigcap_{\beta \in \Delta} U_{n, \beta} \cap\left[R-\bigcup_{\beta \notin \Delta} F_{n, \beta}\right]$ which is open since $\mathscr{F}_{n}$ is closure preserving.

$\mathscr{V}_{n}=\left\{V_{n, \Delta} \mid \Delta \subset B_{n} ; \Delta\right.$ finite $\}$ can be shown to be a star finite covering of $R$ as in the proof of Theorem 4.1, I $\Rightarrow$ II(i).

Suppose $V_{n, \Delta} \cap F_{n, \beta}^{\prime \prime} \neq \varnothing$. Then $\beta \in \Delta$, and $V_{n, \Delta} \subset U_{n, \beta}$. Thus we have, for $p \in F_{n, \beta}^{\prime \prime}, S\left(p, \mathscr{V}_{n}\right) \subset S\left(F_{n, \beta}^{\prime \prime}, \mathscr{V}_{n}\right) \subset U_{n, \beta}$.

Take $\mathscr{S}=\bigcup_{n=1}^{\infty} ; \mathscr{S}_{n} \mathscr{S}_{n}=\left\{S\left(p, \mathscr{V}_{n}\right) \mid p \in R\right\}$. Then $\mathscr{S}_{n}$ is a symmetric star finite open covering of $R$.

A subcollection

$$
\mathscr{S}^{\prime}=\bigcup_{n=1}^{\infty} \mathscr{S}_{n}^{\prime} ; \quad \mathscr{S}_{n}^{\prime}=\left\{S\left(p, \mathscr{V}_{n}\right) \mid p \in P_{n}\right\}
$$

where $P_{n} \subset R$, refines $\mathscr{U}^{\prime}$.

Thus $\mathscr{S}^{\prime}$ refines $\mathscr{W}$, and $R$ satisfies $\mathrm{I}(\mathrm{i})$.

$\mathrm{I}(\mathrm{i}) \Rightarrow \mathrm{II}(\mathrm{ii}) \Rightarrow \mathrm{II}(\mathrm{iii})$ is obvious.

II(iii) $\Rightarrow$ II(iv). By Lemma 3.2, $\mathscr{U}_{n}$ is star finite. Its sets are open, hence $\mathscr{U}_{n}$ is locally finite as well and II(iv) holds.

II(iv) $\Rightarrow$ II(vi). Let $\mathscr{W}$ be an arbitrary open covering of $R$. Since $R$ is regular, $\mathscr{W}$ has an open refinement $\mathscr{V}$ the collection of whose closures refines $\mathscr{W}$.

By hypothesis $\mathscr{V}$ has a refinement $\mathscr{U}^{\prime}=\bigcup_{n=1}^{\infty} \mathscr{U}_{n}^{\prime}$, with $\mathscr{U}_{n}^{\prime}=\left\{U_{n}(p) \mid p \in P_{n}\right\}$, where $P_{n} \subset R, \bigcup_{n=1}^{\infty} P_{n}=R$, which is a subcollection of $\mathscr{U}=\bigcup_{n=1}^{\infty} \mathscr{U}_{n}, \mathscr{U}_{n}=\left\{U_{n}(p) \mid\right.$ $p \in R\}$, where $\mathscr{U}_{n}$ is a symmetric locally finite collection of neighborhoods of points $p$ in $R$.

By Lemma 3.3, for each $n$ the collection $\mathrm{Cl}\left(\mathscr{U}_{n}\right)=\left\{\mathrm{Cl}\left(U_{n}(p)\right) \mid p \in R\right\}$ is star finite.

Put

$$
V_{n, \Delta}=\bigcap_{p \in \Delta} \mathrm{Cl}\left(U_{n}(p)\right) \cap\left[\bigcap_{p \notin \Delta} \mathrm{Cl}\left(R-U_{n}(p)\right)\right]
$$

for $\Delta$ a finite subset of $R^{\prime}$ where $R^{\prime}$ is a subset of $R$ such that $p, q \in R^{\prime}, p \neq q$ implies $U_{n}(p) \neq U_{n}(q)$. Then $p \notin \Delta$ means $p \in R^{\prime}-\Delta$. Then as in the proof of Theorem 4.1, $\mathrm{II}(\mathrm{iv}) \Rightarrow \mathrm{II}(\mathrm{v}), \mathscr{V}_{n}=\left\{V_{n, \Delta} \mid \Delta\right.$ a finite subset of $\left.R^{\prime}\right\}$ is a closed, star finite, locally finite covering of $R$ and $S\left(p, \mathscr{V}_{n}\right) \subset \mathrm{Cl}\left(U_{n}(p)\right)$. Set

$$
\mathscr{S}=\bigcup_{n=1}^{\infty} \mathscr{S}_{n}, \quad \mathscr{S}_{n}=\left\{S\left(p, \mathscr{V}_{n}\right) \mid p \in R\right\}
$$


Then $\mathscr{S}_{n}$ is a symmetric locally finite closed covering of $R$, and a subcollection of $\mathscr{S}$, viz.,

$$
\mathscr{S}^{\prime}=\bigcup_{n=1}^{\infty} \mathscr{S}_{n}^{\prime}, \quad \mathscr{S}_{n}=\left\{S\left(p, \mathscr{V}_{n}\right) \mid p \in P_{n}\right\}, \quad P_{n} \subset R
$$

refines $\mathscr{W}$.

For $p \in P_{n}$ implies $S\left(p, \mathscr{V}_{n}\right) \subset \mathrm{Cl}\left(U_{n}(p)\right) \subset \mathrm{Cl}(V) \subset W$ for some $W \in \mathscr{W}$. Thus II(vi) holds.

II(vi) $\Rightarrow$ I. Let $\mathscr{W}$ be an arbitrary open covering of $R$. By hypothesis $\mathscr{W}$ has a refinement $\mathscr{I}^{\prime}=\bigcup_{n=1}^{\infty} \mathscr{I}_{n}^{\prime}$, with $\mathscr{I}_{n}^{\prime}=\left\{U_{n}(p) \mid p \in P_{n}\right\}, P_{n} \subset R, \bigcup_{n=1}^{\infty} P_{n}=R$, which is a subcollection of $\mathbb{I}=\bigcup_{n=1}^{\infty} \mathbb{I}_{n}, \mathbb{I}_{n}=\left\{U_{n}(p) \mid p \in R\right\}, \mathbb{I}_{n}$ a symmetric locally finite collection of closed sets.

Set $V_{n}(p)=R-\bigcup\left\{U_{n}(q) \mid p \notin U_{n}(q)\right\}$. Then as in the proof of Theorem 3.1 $\mathrm{II}(\mathrm{vi}) \Rightarrow \mathrm{I}, \mathscr{V}_{n}=\left\{V_{n}(p) \mid p \in R\right\}$ is an open star finite covering of $R$ and $V_{n}(p) \subset U_{n}(p)$.

Put $\mathscr{V}=\bigcup_{n=1}^{\infty} \mathscr{V}_{n}$. Then a subcollection of $\mathscr{V}, \mathscr{V}^{\prime}=\bigcup_{n=1}^{\infty} \mathscr{V}_{n}^{\prime}, \mathscr{V}_{n}^{\prime}=\left\{V_{n}(p) \mid\right.$ $\left.p \in P_{n}\right\}, P_{n} \subset R$, refines $\mathscr{W}$. For $p \in P_{n}$ implies $p \in V_{n}(p) \subset U_{n}(p) \subset W$ for some $W \in \mathscr{W}$. Thus $R$ is completely paracompact, and I holds.

$\mathrm{II}(\mathrm{v}) \Rightarrow \mathrm{II}(\mathrm{vi})$. Let $\mathscr{W}$ be an arbitrary open covering of $R$. Since $R$ is regular, $R$ has an open covering $\mathscr{V}$ the collection of closures of whose elements refines $\mathscr{W}$. By hypothesis $\mathscr{V}$ has a refinement $\mathscr{U}^{\prime}=\bigcup_{n=1}^{\infty} \mathscr{U}_{n}^{\prime}$ with

$$
\mathscr{U}_{n}^{\prime}=\left\{U_{n}(p) \mid p \in P_{n}\right\}, \quad P_{n} \subset R, \quad \bigcup_{n=1}^{\infty} P_{n}=R,
$$

which is a subcollection of

$$
\mathscr{U}=\bigcup_{n=1}^{\infty} \mathscr{U}_{n} ; \quad \mathscr{U}_{n}=\left\{U_{n}(p) \mid p \in R\right\},
$$

a locally finite collection of sets such that $q \in \mathrm{Cl}\left(U_{n}(p)\right)$ implies $p \in \mathrm{Cl}\left(U_{n}(q)\right)$.

Then $\mathrm{Cl}\left(\mathscr{U}_{n}\right)=\left\{\mathrm{Cl}\left(U_{n}(p)\right) \mid p \in R\right\}$ is locally finite and symmetric, and a subcollection of $\mathrm{Cl}(\mathscr{U})=\bigcup_{n=1}^{\infty} \mathrm{Cl}\left(\mathscr{U}_{n}\right)$,

$$
\mathrm{Cl}\left(\mathscr{U}^{\prime}\right)=\bigcup_{n=1}^{\infty} \mathrm{Cl}\left(\mathscr{U}_{n}^{\prime}\right), \quad \mathrm{Cl}\left(\mathscr{U}_{n}^{\prime}\right)=\left\{U_{n}(p) \mid p \in P_{n}\right\}, \quad P_{n} \subset R,
$$

refines $\mathscr{W}$. For $p \in P_{n}$ implies $\mathrm{Cl}\left(U_{n}(p)\right) \subset \mathrm{Cl}(V) \subset W$ for some $W \in \mathscr{W}$. Hence II(vi) holds.

$\mathrm{II}(\mathrm{vi}) \Rightarrow \mathrm{II}(\mathrm{v})$. This is obvious.

$\mathrm{II}(\mathrm{i}) \Rightarrow \mathrm{II}($ vii $) \Rightarrow \mathrm{II}($ viii) $\Rightarrow \mathrm{II}(\mathrm{ix})$ is evident.

II(ix) $\Rightarrow$ III. As in the proof of Theorem 3.1 II(ix) $\Rightarrow$ III, $\mathscr{U}_{n}$ is star countable.

III $\Rightarrow$ I. Let $\mathscr{W}$ be an arbitrary open covering of $R$. Then $\mathscr{W}$ has an open refinement

$$
\mathscr{U}^{\prime}=\bigcup_{n=1}^{\infty} \mathscr{U}_{n}^{\prime} ; \quad \mathscr{U}_{n}^{\prime}=\left\{U_{n, \beta} \mid \beta \in B_{n}^{\prime}\right\}, \quad B_{n}^{\prime} \subset B_{n},
$$

which is a subcollection of $\mathscr{U}=\bigcup_{n=1}^{\infty} \mathscr{U}_{n}, \mathscr{U}_{n}=\left\{U_{n, \beta} \mid \beta \in B_{n}\right\}, \mathscr{U}_{n}$ a star countable open covering of $R$. 
For each $n,\left\{U_{n, \beta} \mid U_{n, \beta} \subset \bigcup_{m=1}^{\infty} S^{m}\left(U_{n, \gamma}, \mathscr{U}_{n}\right)\right\}$ is countable. Let us denote each set in such a collection by $U_{n, \beta(i, \gamma)} ; i=1,2, \ldots ; \gamma \in \Gamma_{n}$, and put $U_{n}^{(\gamma)}=\bigcup_{i=1}^{\infty} U_{n, \beta(i, \gamma)}$; where we take a subset $\Gamma_{n}$ of $B_{n}$ so that $U_{n}^{(\gamma)} \cap U_{n}^{(\delta)}=\varnothing$ for $\gamma, \delta \in \Gamma_{n}, \gamma \neq \delta$.

If we choose one set $U_{n, \beta(i, \gamma)}$ for each $\gamma \in \Gamma_{n}$, this will be a discrete collection, and countably many such collections make up $\mathscr{U}_{n}$. Thus $\mathscr{U}=\bigcup_{n=1}^{\infty} \mathscr{U}_{n}$ is $\sigma$-discrete, $\mathscr{U}^{\prime}$ is $\sigma$-discrete also and $R$ is paracompact.

Since $U_{n}^{(\gamma)}$ is closed in $R$ it is paracompact, hence there exists a closed covering of $U_{n}^{(\gamma)},\left\{F_{n, \beta(i, \gamma)} \mid i=1,2, \ldots\right\}$ such that $F_{n, \beta(i, \gamma)} \subset U_{n, \beta(i, \gamma)} ; i=1,2, \ldots$

Since $R$ itself is paracompact, there exists a closed covering $\left\{F_{n, \beta}^{\prime} \mid \beta \in B_{n}^{\prime}\right.$; $n \in\{1,2, \ldots\}\}$ of $R$ such that $F_{n, \beta}^{\prime} \subset U_{n, \beta}$ for every $\beta \in B_{n}^{\prime}, n \in\{1,2, \ldots\}$.

For every $n$ and $\gamma \in \Gamma_{n}$, we form a countable closed covering $\mathscr{F}_{n, y}$ of $U_{n}^{(\gamma)}$ as follows:

$$
\begin{aligned}
F_{n, \beta(i, \gamma)}^{n} & =F_{n, \beta(i, \gamma)} & & \text { for } \beta(i, \gamma) \notin B_{n}^{\prime}, \\
& =F_{n, \beta(i, \gamma)} \cup F_{n, \beta(i, \gamma)}^{\prime} & & \text { for } \beta(i, \gamma) \in B_{n}^{\prime} .
\end{aligned}
$$

Then $F_{n, \beta(i, \gamma)}^{n} \subset U_{n, \beta(i, \gamma)}$ for $U_{n, \beta(i, \gamma)} \subset U_{n}^{(\gamma)}$, and

$$
\bigcup\left\{F_{n, \beta(i, \gamma)}^{\prime \prime} \mid \beta(i, \gamma) \in B_{n}^{\prime} ; \gamma \in \Gamma_{n}, n=1,2, \ldots\right\}=R .
$$

Since $U_{n}^{(\gamma)}$ is normal, we can construct open sets $G_{n, \beta(i, \gamma)}^{(k)} ; i=1,2, \ldots ; k=i$, $i+1, \ldots$, such that

$$
F_{n, \beta(i, \gamma)}^{\prime \prime} \subset G_{n, \beta(i, \gamma)}^{(k)} \subset \mathrm{Cl}\left(G_{n, \beta(i, \gamma)}^{(k)}\right) \subset G_{n, \beta(i, \gamma)}^{(k+1)} \subset U_{n, \beta(i, \gamma)}
$$

Set $X_{n, \gamma, k}=\bigcup_{i=1}^{k} G_{n, \beta(i, \gamma)}^{(k)}$.

Then $U_{n}^{(\gamma)}=\bigcup_{k=1}^{\infty} X_{n, \gamma, k}$, and $\mathrm{Cl}\left(X_{n, \gamma, k}\right) \subset X_{n, \gamma, k+1} ; k=1,2, \ldots$

To see this, let $x \in \mathrm{Cl}\left(X_{n, \gamma, k}\right)=\bigcup_{i=1}^{k} \mathrm{Cl}\left(G_{n, \beta(i, \gamma)}^{(k)}\right)$. Then $x \in \mathrm{Cl}\left(G_{n, \beta(i, \gamma)}^{(k)}\right)$ for some $i \leqq k$. This implies $x \in G_{n, \beta(i, y)}^{(k+1)}$ for $i \leqq k$. Hence

$$
x \in \bigcup_{i=1}^{k+1} G_{n, \beta(i, \gamma)}^{(k+1)}=X_{n, \gamma, k+1}
$$

Now let us set

$$
\begin{aligned}
& H_{n, \gamma, k}=X_{n, \gamma, k}-\mathrm{Cl}\left(X_{n, \gamma, k-3}\right), \\
& K_{n, \gamma, k}=\mathrm{Cl}\left(X_{n, \gamma, k}\right)-X_{n, \gamma, k-1}, \quad k=1,2, \ldots
\end{aligned}
$$

where $X_{n, y, k}=\varnothing$ for $k \leqq 0$.

Then $H_{n, y, k}$ is open and $K_{n, \gamma, k}$ is closed. Moreover we have:

$$
\begin{aligned}
K_{n, \gamma, k} & \subset H_{n, \gamma, k+1}, \\
\bigcup_{k=1}^{\infty} K_{n, \gamma, k} & =U_{n}^{(\gamma)}, \\
H_{n, \gamma, k} \cap H_{n, \gamma, j} & =\varnothing \text { for }|k-j| \geqq 3 .
\end{aligned}
$$


Since $K_{n, \gamma, k}=\mathrm{Cl}\left(X_{n, \gamma, k}\right)-X_{n, \gamma, k-1} \subset \mathrm{Cl}\left(X_{n, \gamma, k}\right)$ then

$$
\begin{aligned}
K_{n, \gamma, k} & =\operatorname{Cl}\left(X_{n, \gamma, k}\right) \cap K_{n, \gamma, k} \\
& =\operatorname{Cl}\left(\bigcup_{i=1}^{k} G_{n, \beta(i, \gamma)}^{(k)}\right) \cap K_{n, \gamma, k} \\
& =\bigcup_{i=1}^{k} \operatorname{Cl}\left(G_{n, \beta(i, \gamma)}^{(k)}\right) \cap K_{n, \gamma, k} .
\end{aligned}
$$

Thus the system of closed sets

$$
\left\{K_{n, \gamma, k} \cap \mathrm{Cl}\left(G_{n, \beta(i, \gamma)}^{(k)}\right) \mid i=1,2, \ldots, k ; k=1,2, \ldots\right\}
$$

forms a covering of $U_{n}^{(\gamma)}$, and therefore

$$
\left\{H_{n, \gamma, k+1} \cap G_{n, \beta(i, \gamma)}^{(k+1)} \mid i=1,2, \ldots, k ; k=1,2, \ldots\right\}
$$

is an open covering of $U_{n}^{(\gamma)}$, since

$$
K_{n, \gamma, k} \cap \mathrm{Cl}\left(G_{n, \beta(i, \gamma)}^{(k)}\right) \subset H_{n, \gamma, k+1} \cap G_{n, \beta(i, \gamma)}^{(k+1)}
$$

by (1) and (2). Moreover, because of (4)

$$
\left\{H_{n, \gamma, k+1} \cap G_{n, \beta(i, y)}^{(k+1)} \mid i=1,2, \ldots, k ; k=1,2, \ldots\right\}
$$

is star finite.

Now, put $H_{n, \gamma, k}^{(j)}=X_{n, \gamma, k}-\mathrm{Cl}\left(X_{n, \gamma, k-(2+j)}\right), j=1,2, \ldots$ Then by a similar discussion, for each $(n, j)$

$$
\mathscr{W}_{n, j}=\left\{H_{n, \gamma, k+1}^{(j)} \cap G_{n, \beta(i, \gamma)}^{(k+1)} \mid i=1,2, \ldots, k ; k=1,2, \ldots ; \gamma \in \Gamma_{n}\right\}
$$

is a star finite open covering of $R$.

Define a subcollection $\mathscr{W}_{n, j}^{\prime}$ of $\mathscr{W}_{n, j}$ by

$$
\mathscr{W}_{n, j}^{\prime}=\left\{H_{n, \gamma, k+1}^{(j)} \cap G_{n, \beta(i, \gamma)}^{(k+1)} \mid \beta(i, \gamma) \in B_{n}^{\prime}\right\}
$$

Then $\bigcup_{n, j=1}^{\infty} \mathscr{W}_{n, j}^{\prime}$ is a covering of $R$ and refines $\mathscr{W}$. For let $x \in F_{n, \beta(i, \gamma)}^{n}$ and $\beta(i, \gamma) \in B_{n}^{\prime}$; then $x \in X_{n, \gamma, k+1}$ for every $k \geqq i$. Hence $x \in H_{n, \gamma, k+1}^{(j)}$ for $j \geqq k-1$ because for such $j, H_{n, \gamma, k+1}^{(j)}=X_{n, \gamma, k+1}$. Thus $\mathrm{I}$ is proved.

Morita [7, Corollary p. 66] has proved that for a regular connected space $R$, the star finite property is equivalent to the Lindelöf property. The following theorem extends this result.

THEOREM 5.2. If $R$ is a regular space, and each of its connected components is open, then complete paracompactness is equivalent to the star finite property in $R$. If the collection of components is countable, then complete paracompactness is equivalent to the Lindelöf property in $R$.

Proof. Since for any regular space the Lindelöf property $\Rightarrow$ the star finite property $\Rightarrow$ complete paracompactness, we need only show that under the 
hypothesis the reverse implications hold. Let us assume $R$ is completely paracompact. By hypothesis, $R=\bigcup\left\{A_{\alpha} \mid \alpha \in \mathscr{A}\right\}, A_{\alpha}$ connected, each $A_{\alpha}$ open and closed in $R$, and $A_{\alpha} \cap A_{\beta}=\varnothing$ for $\alpha \neq \beta$.

Let $\mathscr{V}$ be an arbitrary open covering of $R$. Since $R$ is completely paracompact, $\mathscr{V}$ has an open refinement $\mathscr{U}^{\prime}$ which is subcollection of $\mathscr{U}=\bigcup_{n=1}^{\infty} \mathscr{U}_{n}, \mathscr{U}_{n}=$ $\left\{U_{n, \beta} \mid \beta \in B_{n}\right\}$ where $\mathscr{U}_{n}$ is a star finite open covering of $R$.

Consider the collection $\mathscr{U}_{n}^{\alpha}=\left\{U_{n, \beta} \cap A_{\alpha} \mid \beta \in B_{n}\right\}$. Since this collection is star finite, $A_{\alpha}$ may be written as the union of disjoint open and closed sets, each of which is the union of countably many elements of $\mathscr{U}_{n}^{\alpha}$. Since $A_{\alpha}$ is connected, there can be only one such set. Hence $\mathscr{U}_{n}^{\alpha}$ is countable for each $n$ and $\alpha$.

It follows that the collection $\left\{U_{n, \beta} \cap A_{\alpha} \mid \beta \in B_{n} ; n=1,2, \ldots\right\}$ is countable for each $\alpha \in \mathscr{A}$. If $\mathscr{A}$ is countable, clearly $\mathscr{U}$ is countable, as is $\mathscr{U}^{\prime}$. For each $U \in \mathscr{U}^{\prime}$ there exists a $V \in \mathscr{V}$ such that $U \subset V$. The collection of all such elements $V$ is a countable subcovering of $\mathscr{V}$, hence $R$ has the Lindelöf property.

Now we assume $\mathscr{A}$ is uncountable. The collection $\left\{U \cap A_{\alpha} \mid U \in \mathscr{U}^{\prime}\right\}$ is a countable open covering of $A_{\alpha}$. Since $A_{\alpha}$ is a closed subset of a completely paracompact space, $A_{\alpha}$ is completely paracompact, hence paracompact. Then by a theorem of Morita [7, Theorem 3] $\left\{U \cap A_{\alpha} \mid U \in \mathscr{U}^{\prime}\right\}$ has a countable open star finite refinement, $\left\{V_{\alpha, \beta} \mid \beta \in B_{\alpha}\right\}$. Since $A_{\alpha}$ is open in $R, V_{\alpha, \beta}$ is open in $R$. Then $\left\{V_{\alpha, \beta} \mid \beta \in B_{\alpha} ; \alpha \in \mathscr{A}\right\}$ is star finite and refines $\mathscr{U}^{\prime}$, hence refines $\mathscr{V}$. Thus $R$ has the star finite property.

NoTE. If $R$ is locally connected, then each of its connected components is open.

Michael's [6] example shows that the cartesian product of two completely paracompact spaces, or even the product of a completely paracompact space with a strongly metrizable space, need not be completely paracompact. However, we can prove the following:

THEOREM 5.3. Let $A$ be a completely paracompact space and let $B$ be a space which is compact and regular. Then $A \times B$ is completely paracompact.

Proof. First of all, $A \times B$ is regular, since both $A$ and $B$ are regular.

Let $\mathscr{W}$ be an arbitrary open covering of $A \times B$. Let $a \in A$. Then each point of $a \times B$ is contained in an open set of the form $U \times V, U$ open in $A, V$ open in $B$, such that $U \times V \subset W$ for some $W \in \mathscr{W}$.

For each fixed point $a \in A$, the set of all such $V$ 's is an open covering of $B$. Since $B$ is compact, a finite number of them, say $V_{a, 1}, V_{a, 2}, \ldots, V_{a, k(a)} \operatorname{cover} B$.

To each $V_{a, i}$ there corresponds a $U_{a, i}$ for which $U_{a, i} \times V_{a, i} \subset W$ for some $W \in \mathscr{W}$.

Put $U_{a}=\bigcap_{i=1}^{k(a)} U_{a, i}$. Then $\mathscr{U}=\left\{U_{a} \mid a \in A\right\}$ is an open covering of $A$. Since $A$ is completely paracompact, $\mathscr{U}$ has a refinement

$$
\mathscr{R}^{\prime}=\bigcup_{n=1}^{\infty} \mathscr{R}_{n}^{\prime}, \quad \mathscr{R}_{n}^{\prime}=\left\{R_{n, \beta} \mid \beta \in B_{n}^{\prime}\right\}, \quad B_{n}^{\prime} \subset B_{n},
$$


which is a subcollection of $\mathscr{R}=\bigcup_{n=1}^{\infty} \mathscr{R}_{n}, \mathscr{R}_{n}=\left\{R_{n, \beta} \mid \beta \in B_{n}\right\} ; \mathscr{R}_{n}$ an open star finite covering of $A$.

For each $n$ we form an open covering of $A \times B$ as follows: For each $\beta \in B_{n}^{\prime}$, $R_{n, \beta} \subset U_{a}$ for some $\alpha \in A$. Set

$$
S_{n, \beta, i}=R_{n, \beta} \times V_{a, i}, \quad i=1,2, \ldots, k(a)
$$

and

$$
S_{n, \beta}=R_{n, \beta} \times B \text { for } \beta \in B_{n}-B_{n}^{\prime} .
$$

For each $n$, the collection $\mathscr{S}_{n}$ of these sets is an open star finite covering of $A \times B$, by the star finiteness of $\mathscr{R}_{n}$. Moreover a subcollection of $\mathscr{S}=\bigcup_{n=1}^{\infty} \mathscr{S}_{n}$ refines $\mathscr{W}$. Clearly

$$
\left\{S_{n, \beta, i} \mid i=1,2, \ldots, k(a) ; \beta \in B_{n}^{\prime} ; n=1,2, \ldots\right\}
$$

covers $A \times B$.

$$
S_{n, \beta, i}=R_{n, \beta} \times V_{a, i} \subset U_{a} \times V_{a, i} \subset U_{a, i} \times V_{a, i} \subset W
$$

for some $W \in \mathscr{W}$, and $A \times B$ is completely paracompact.

VI. Dimension. There are three basic ways in which the dimension of a topological space is defined: covering dimension, or Lebesgue dimension, (dim) which is the dimension defined by finite open coverings; small inductive dimension, or Menger-Urysohn dimension, (ind) the dimension defined inductively in terms of neighborhoods of points, and large inductive dimension (Ind), the dimension defined inductively in terms of neighborhoods of closed sets.

Katětov [3] and Morita [8, Theorem 8.6] have proved that for a metric space $R$, $\operatorname{dim} R=$ Ind $R$, and it is obvious that ind $R \leqq$ Ind $R$ generally holds. Roy [13] has shown that in a metric space the reverse inequality does not necessarily hold.

However for a strongly metrizable space the three basic definitions of dimension are equivalent. Zarelua [19] has proved this, and in this section we give a proof different from his.

THEOREM 6.1. For a metric space $R$ and an arbitrary property $P$ of families of subsets of $R$, the following are equivalent:

(i) $R$ has a basis $\mathscr{U}=\bigcup_{n=1}^{\infty} \mathscr{U}_{n}, \mathscr{U}_{n}=\left\{U_{n, \beta} \mid \beta \in B_{n}\right\}, \mathscr{U}_{n}$ a covering of $R$, where for every $n$ the collection $\mathscr{U}_{n}$ has property $P$.

(ii) Every open covering of $R$ has a refinement $\mathscr{U}^{\prime}$ which is a subcollection of $\mathscr{U}=\bigcup_{n=1}^{\infty} \mathscr{U}_{n}, \mathscr{U}_{n}=\left\{U_{n, \beta} \mid \beta \in B_{n}\right\}, \mathscr{U}_{n}$ an open covering of $R$, where for every $n$, the collection $\mathscr{U}_{n}$ has property $P$.

Proof. (i) $\Rightarrow$ (ii) is obvious.

(ii) $\Rightarrow$ (i). For every $n, \mathscr{V}_{n}=\{S(p, 1 / n) \mid p \in R\}$ is an open covering of $R$. By hypothesis, $\mathscr{V}_{n}$ has a refinement $\mathscr{U}_{n}^{\prime}$ which is a subcollection of $\mathscr{U}_{n}=\bigcup_{m=1}^{\infty} \mathscr{U}_{n, m}$, where $\mathscr{U}_{n, m}$ covers $R$ and has property $P$. 
Then $\mathscr{I}=\bigcup_{n, m=1}^{\infty} \mathbb{I}_{n, m}$ is a basis for $R$, where $\mathscr{U}_{n, m}$ covers $R$ and has property $P$.

If, for example, property $P$ is that $\mathbb{I}_{n}$ is a star finite collection we have the following:

COROLlaRY. For a metric space $R$ complete paracompactness is equivalent to strong metrizability.

This has been proved by Zarelua [19, Lemma 3].

We can apply this result to prove an interesting theorem in dimension theory, if we take property $P$ to be that $\mathscr{I}_{n}$ is a locally finite collection of open sets such that $\operatorname{dim}(\operatorname{Fr}(U)) \leqq m-1$ for every $U \in \mathbb{U}_{n}$.

First we state a theorem that is due to Morita [8, Theorem 8.7].

THEOREM 6.2 (MORITA). Let $R$ be a nietric space. Then $\operatorname{dim} R \leqq m$ if and only if there exists a countable collection of locally finite open coverings $\mathscr{U}_{n}$ of $R$ such that II $=\bigcup_{n=1}^{\infty} \mathscr{I I}_{n}$ is a basis for $R$ and $\operatorname{dim}(\operatorname{Fr}(U)) \leqq m-1$ for every $U \in \mathscr{U}$.

THEOREM 6.3. Let $R$ be a metric space. Then $\operatorname{dim} R \leqq m$ if and only if every open covering of $R$ has an open refinement $\mathscr{I}=\bigcup_{n=1}^{\infty} \mathscr{U}_{n}$, where $\mathscr{U}_{n}$ is locally finite and $\operatorname{dim}(\operatorname{Fr}(U)) \leqq m-1$ for every $U \in \mathscr{U}$.

Proof. We may apply Theorems 6.1 and 6.2.

In proving "if", we may extend $\mathscr{U}_{n}$ to a covering of $R$, as demanded in Theorem 6.1 without spoiling its properties by adjoining $R$ to it.

Applying Theorem 6.3, we obtain an alternate proof of a theorem of Zarelua [19, Corollary 2, p. 1536].

THEOREM 6.4. Let $R$ be a strongly metrizable space. Then ind $R \leqq m$ implies $\operatorname{dim} R \leqq m$.

Proof. We proceed by induction and assume the theorem true for $m-1$.

Let $\mathscr{W}$ be an arbitrary open covering of $R$. Since ind $R \leqq m$, for every $p \in R$ there exists an open neighborhood $V(p)$ such that

(1) $p \in V(p) \subset W$ for some $W \in \mathscr{W}$ and ind $(\operatorname{Fr}(V(p))) \leqq m-1$.

Then $\{V(p) \mid p \in R\}$ refines $\mathscr{W}$. Since $R$ is strongly metrizable, $R$ has an open basis $\mathscr{U}=\bigcup_{n=1}^{\infty} \mathscr{U}_{n}$, where $\mathscr{U}_{n}$ is a star finite covering of $R$.

We can suppose that $\mathscr{U}_{n}$ consists of open sets $U_{n, \gamma, i} ; \gamma \in \Gamma_{n} ; i=1,2, \ldots$ such that $U_{n, \gamma, i} \cap U_{n, \delta, j}=\varnothing ; \gamma \neq \delta$.

Set $U_{n, \gamma}=\bigcup_{i=1}^{\infty} U_{n, y, i}$. Then $U_{n, \gamma}$ is both open and closed in $R$ as $\mathscr{U}_{n}$ was an open covering of $R$.

Now an element $U_{n, \gamma, i}$ of $\mathscr{U}_{n}$ may perhaps be contained in some $V(p)$ satisfying (1). If so, we choose one such $V(p)$ and denote it by $V_{n, r, i}$; if no such $V(p)$ exists, put $V_{n, \gamma, i}=\varnothing$.

Set $X_{n, \gamma, i}=U_{n, \gamma} \cap V_{n, \gamma, i}$. Then

$$
\operatorname{Fr}\left(X_{n, y, i}\right)=\operatorname{Fr}\left(U_{n, y} \cap V_{n, \gamma, i}\right) \subset U_{n, \gamma} \cap \operatorname{Fr}\left(V_{n, \gamma, i}\right)
$$

which implies ind $\left(\operatorname{Fr}\left(X_{n, \gamma, i}\right)\right) \leqq m-1$. 
By the induction assumption and since every subspace of a strongly metrizable space is strongly metrizable, we have

(2) $\operatorname{dim}\left(\operatorname{Fr}\left(X_{n, \gamma, i}\right)\right) \leqq m-1$, and $X_{n, \gamma, i} \subset V_{n, \gamma, i}$.

For fixed $n$ and $i$, the collection $\left\{X_{n, \gamma, i} \mid \gamma \in \Gamma_{n}\right\}$ is discrete. Thus we get countably many discrete collections which together form an open covering refining $\mathscr{W}$. Then by Theorem 6.3 we have $\operatorname{dim} R \leqq m$.

Corollary 6.5 (Zarelua). In a strongly metrizable space $R$, Ind $R=$ ind $R$ $=\operatorname{dim} R$.

VII. Some mapping theorems. It seems to be unknown whether the star finite property is invariant under a closed continuous mapping $f$ of a space $R$ onto a space $S$, such that $f^{-1}(p)$ is compact for every $p \in S$, i.e., a perfect (or proper) mapping.

Ponomarev [12] has proved that if $R$ is a Hausdorff space having the star finite property, and $f$ is a (multi-valued) open and closed continuous mapping of $R$ onto $S$, such that the images $f(x)$, and inverse images $f^{-1}(p)$ are compact for every $x \in R, p \in S$, then $S$ has the star finite property.

In this section, we prove some mapping theorems by imposing the condition that $f$ be monotone, i.e., $f^{-1}(p)$ is connected for each $p \in S$.

THEOREM 7.1. Let $f$ be a perfect, monotone mapping of a regular topological space $R$ having the star finite property onto a topological space $S$. Then $S$ is a regular space having the star finite property.

Proof. Since $R$ is regular and paracompact, it is normal, thus $S$ is normal by a theorem of Whyburn [18, Theorem 9]. Now we need to show that $S$ has the star finite property.

Let $\mathscr{W}$ be an arbitrary open covering of $S$. Since $S$ is paracompact [5, Corollary 1], $\mathscr{W}$ has an open $\Delta$-refinement $\mathscr{U}=\left\{U_{\alpha} \mid \alpha \in A\right\}$.

$\mathscr{U}^{\prime}=\left\{f^{-1}\left(U_{\alpha}\right) \mid \alpha \in A\right\}$ is an open covering of $R$. Since $R$ has the star finite property, $\mathscr{U}^{\prime}$ has an open star finite refinement $\mathscr{V}=\left\{V_{\beta} \mid \beta \in B\right\}$.

Since $f^{-1}(p)$ is compact, only finitely many elements of $\mathscr{V}$ meet $f^{-1}(p)$, say $\left\{V_{\beta(i, p)} \mid i=1,2, \ldots, n(p)\right\}$.

For $X \subset R$, write $X_{0}=f^{-1}(S-f(R-X))$; this is an inverse set contained in $X$, and $f\left(X_{0}\right)=S-f(R-X)$, so that $f\left(X_{0}\right)$ and $X_{0}$ are open if $X$ is open (because $f$ is closed and continuous). Thus

$$
\mathscr{M}=\left\{f\left[\left(\bigcup_{i=1}^{n(p)} V_{\beta(i, p)}\right)_{0}\right] \mid p \in S\right\}
$$

is an open covering of $S$.

(1) $\mathscr{M}$ refines $\mathscr{W}$.

$\left(\bigcup_{i=1}^{n(p)} V_{\beta(i, p)}\right)_{0} \subset \bigcup_{i=1}^{n(p)} V_{\beta(i, p)}$, hence

$$
f\left[\left(\bigcup_{i=1}^{n(p)} V_{B(i, p)}\right)_{0}\right] \subset f\left(\bigcup_{i=1}^{n(p)} V_{B(i, p)}\right) .
$$


Moreover $f\left(\bigcup_{i=1}^{n(p)} V_{\beta(i, p)}\right) \subset S(p, \mathbb{M}) \subset W$ for some $W \in \mathscr{W}$.

(2) $\mathscr{M}$ is star countable.

First, we show that

$$
\left\{\bigcup_{i=1}^{n(p)} V_{R(i, p)} \mid p \in S\right\}=\left\{S\left(f^{-1}(p), \mathscr{Y}\right) \mid p \in S\right\}
$$

is star countable.

Let $V_{\beta(i, p)} \in\left\{V_{\beta(i, p)} \mid i=1,2, \ldots, n(p)\right\}$. Since $f^{-1}(p)$ is connected, for every $V_{\beta(j, p)} \in\left\{V_{\beta(i, p)} \mid i=1,2, \ldots, n(p)\right\}$ there exists a $k$ such that $V_{\beta i j, p)} \subset S^{k}\left(V_{\beta(i, p),}, \mathscr{V}\right)$.

Suppose $S\left(f^{-1}(p), \mathscr{V}\right) \cap S\left(f^{-1}(q), \mathscr{V}\right) \neq \varnothing$. Then every $V_{\beta(i, q)}$ in $S\left(f^{-1}(q), \mathscr{V}\right)$ must be an element of the countable family of sets of $\mathscr{V}$ which are contained in $S^{n}\left(V_{\beta(i, p)}, \mathscr{Y}^{\wedge}\right)$ for some $n$. Only countably many finite collections can be made up from sets taken from this countable family, thus $S\left(f^{-1}(q), \mathscr{V}\right)$ is an element of a countable family, and $\left\{S\left(f^{-1}(p), \mathscr{V}\right) \mid p \in S\right\}$ is star countable.

It follows that $\left\{\left(\bigcup_{i=1}^{n(p)} V_{\beta(i, p)}\right)_{0} \mid p \in S\right\}$ is star countable, and therefore $\mathscr{M}$ is star countable. By Theorem 3.1 this implies that $S$ has the star finite property, thus the theorem is proved.

THEOREM 7.2. Let $f$ be an open continuous monotone mapping of a regular space $R$ having the star finite property onto a regular space $S$. Then $S$ has the star finite property.

Proof. Let $\mathscr{W}=\left\{W_{\alpha} \mid \alpha \in A\right\}$ be an arbitrary open covering of $S$. Then $\mathscr{W}^{\prime \prime}$ $=\left\{f^{-1}\left(W_{\alpha}\right) \mid \alpha \in A\right\}$ is an open covering of $R . \mathscr{W}^{\prime}$ has an open star finite refinement $\mathscr{V}=\left\{V_{\beta} \mid \beta \in B\right\}$.

Put $\mathscr{V}^{\prime}=\left\{f\left(V_{\beta}\right) \mid \beta \in B\right\}$. Then $\mathscr{V}^{\prime}$ is an open covering of $S$, it refines $\mathscr{W}$, and it is star countable.

First it is obvious that $\mathscr{V}^{\prime}$ refines $\mathscr{W}$.

Now we show that $\mathscr{V}^{\prime}$ is star countable. Suppose $f\left(V_{\beta}\right) \cap f\left(V_{\alpha}\right) \neq \varnothing, \alpha, \beta \in B$. Then there exists an $r \in f\left(V_{\beta}\right) \cap f\left(V_{\alpha}\right)$, and $f^{-1}(r) \cap V_{\beta} \neq \varnothing ; f^{-1}(r) \cap V_{\alpha} \neq \varnothing$. Since $f^{-1}(r)$ is connected, this means that for some positive integer $n$, $V_{\alpha} \subset S^{n}\left(V_{\beta}, \mathscr{V}\right)$. Since $\mathscr{V}$ is star finite, $V_{\alpha}$ is an element of a countable family, thus

$$
\left\{f\left(V_{\alpha}\right) \mid f\left(V_{\alpha}\right) \cap f\left(V_{\beta}\right) \neq \varnothing ; \alpha \in B\right\}
$$

is a countable family also. Thus $\mathscr{V}^{\prime}$ is star countable, and $S$ has the star finite property.

By a similar argument, we have Theorems 7.3 and 7.4 .

THEOREM 7.3. Let $f$ be an open continuous monotone mapping of a completely paracompact space $R$ onto a regular space $S$. Then $S$ is completely paracompact.

THEOREM 7.4. Let $f$ be an open continuous monotone mapping of a strongly metrizable space $R$ onto a regular space $S$. Then $S$ is strongly metrizable.

THEOREM 7.5. Let $f$ be a closed, continuous mapping of a regular topological 
space $R$ onto a completely paracompact space $S$ such that $f^{-1}(y)$ has the Lindelöf property for every $y \in S$. Then $R$ is completely paracompact.

Proof. Let $\mathscr{O l}$ be an arbitrary open covering of $R$. For every $y \in S$ there exists a countable subcollection, $\mathscr{U}_{y}=\left\{U_{i} \mid i=1,2, \ldots\right\}$ which covers $f^{-1}(y)$. Put

$$
U_{y}=\bigcup_{i=1}^{\infty} U_{i}, \quad V_{y}=S-f\left(R-U_{y}\right) .
$$

Then $V_{y}$ is an open neighborhood of $y$ in $S$ and $f^{-1}\left(V_{y}\right) \subset U_{y} . \mathscr{V}=\left\{V_{y} \mid y \in S\right\}$ is an open covering of $S$. Since $S$ is completely paracompact, $\mathscr{V}$ has an open refinement $\mathscr{W}^{\prime}$ which is a subcollection of $\mathscr{W}=\bigcup_{n=1}^{\infty} \mathscr{W}_{n} ; \mathscr{W}_{n}$ a star open covering of $S$.

Write $\mathscr{W}^{\prime}=\left\{W_{n, \beta}^{\prime} \mid \beta \in B_{n}, n \in\{1,2, \ldots\}\right\}$. For each index $n, \beta$ in the collection $\mathscr{W}^{\prime}$, there exists a $y(n, \beta)$ such that $W_{n, \beta}^{\prime} \subset V_{y(n, \beta)}$. This implies

(1) $f^{-1}\left(W_{n, \beta}^{\prime}\right) \subset f^{-1}\left(V_{y(n, \beta)}\right) \subset U_{y / n, \beta)}$.

The collection

$$
\mathscr{S}_{n}=\left\{f^{-1}\left(W_{n, \beta}^{\prime}\right) \cap U_{i} \mid U_{i} \in \mathscr{U}_{y(n, \beta)} ; W_{n, \beta}^{\prime} \in \mathscr{W}^{\prime}\right\} \cup\left\{f^{-1}\left(W_{n, \beta}\right) \mid W_{n, \beta} \notin \mathscr{W}^{\prime}\right\}
$$

is an open covering of $R$.

Now we must show that $\mathscr{S}_{n}$ is star countable. Since $\mathscr{W}_{n}$ is star finite, clearly $\left\{f^{-1}\left(W_{n, \beta}\right) \mid W_{n, \beta} \in \mathscr{W}_{n}\right\}$ is star finite. Since $\mathscr{U}_{y}$ is a countable collection for every $y \in S$, then $\mathscr{S}_{n}$ is star countable. Put $\mathscr{S}=\bigcup_{n=1}^{\infty} \mathscr{S}_{n}$.

The subcollection $\mathscr{S}^{\prime}$ of $\mathscr{S}$

$$
\mathscr{S}^{\prime}=\left\{f^{-1}\left(W_{n, \beta}^{\prime}\right) \cap U_{i} \mid U_{i} \in \mathscr{U}_{y(n, \beta)} ; W_{n, \beta}^{\prime} \in \mathscr{W}^{\prime}\right\}
$$

covers $R$ and refines $\mathscr{U}$. Hence by Theorem $5.1 R$ is completely paracompact.

COROLlaRY 7.6. If $f$ is a closed continuous mapping of a metric space $R$ onto a completely paracompact space $S$ such that $f^{-1}(y)$ is separable and has a compact frontier for every $y \in S$, then both $R$ and $S$ are strongly metrizable.

Proof. Stone [16, Theorem 1] and Hanai and Morita [2, Theorem 1] have proved that if $f$ is a closed continuous mapping of a metric space $R$ onto a space $S$ such that $f^{-1}(p)$ has a compact frontier for all $p \in S$, then $S$ is metrizable.

By Theorem 6.1, a completely paracompact metric space is strongly metrizable. These results together with Theorem 7.5 prove the corollary.

\section{REFERENCES}

1. J. Ceder, Some generalizations of metric spaces, Pacific J. Math. 11 (1961), 105-125.

2. S. Hanai and K. Morita, Closed mappings and metric spaces, Proc. Japan Acad. 32 (1956), $10-14$.

3. M. Katětov, On the dimension of non-separable spaces. I, Czechoslovak Math. J. 2 (1952), 333-368.

4. E. Michael, A note on paracompact spaces, Proc. Amer. Math. Soc. 4 (1953), 831-838.

5. - Another note on paracompact spaces, Proc. Amer. Math. Soc. 8 (1957), 822-828.

6. - The product of a normal space and a metric space need not be normal, Bull. Amer.

Math. Soc. 69 (1963), 375-376. 
7. K. Morita, Star-finite coverings and the star-finite property, Math. Japon. 1 (1948), 60-68.

8. - - Normal families and dimension theory for metric spaces, Math. Ann. 128 (1954), $350-362$.

9. J. Nagata, A contribution to the theory of metrization, J. Inst. Polytech. Osaka City Univ. Ser. A 8 (1957), 185-192.

10. - - A note on dimension theory for metric spaces, Fund. Math. 45 (1958), 143-181.

11. - On a necessary and sufficient condition of metrizability, J. Inst. Polytech. Osaka City Univ. Ser. A. Math. 1 (1950), 93-100.

12. V. Ponomarev, Proof of the invariance of the star finite property under open perfect mappings, Bull. Acad. Polon. Sci. Sér. Sci. Math. Astronom. Phys. 10 (1962), 425-428.

13. P. Roy, Failure of equivalence of dimension concepts for metric spaces, Bull. Amer. Math. Soc. 68 (1962), 609-613.

14. Yu. Smirnov, On strongly paracompact spaces, Izv. Akad. Nauk. SSSR. Ser. Mat. 20 (1956), 253-274.

15. A. H. Stone, Paracompactness and product spaces, Bull. Amer. Math. Soc. 54 (1948), 977-982.

16. - Metrizability of decomposition spaces, Proc. Amer. Math. Soc. 7 (1956), 690-700.

17. - Universal spaces for some metric uniformities, Quart. J. Math. Oxford Ser. (2) 11 (1960), 105-115.

18. G. T. Whyburn, Open and closed mappings, Duke Math. J. 17 (1950), 69-74.

19. A. Zarelua, On a theorem of Hurewicz, Dokl. Akad. Nauk. SSSR 141 (1961), 777-780= Soviet Math. Dokl. 2 (1961), 1534-1537.

\section{UNIVERSITY OF SAINT THOMAS,}

Houston, TEXAS 